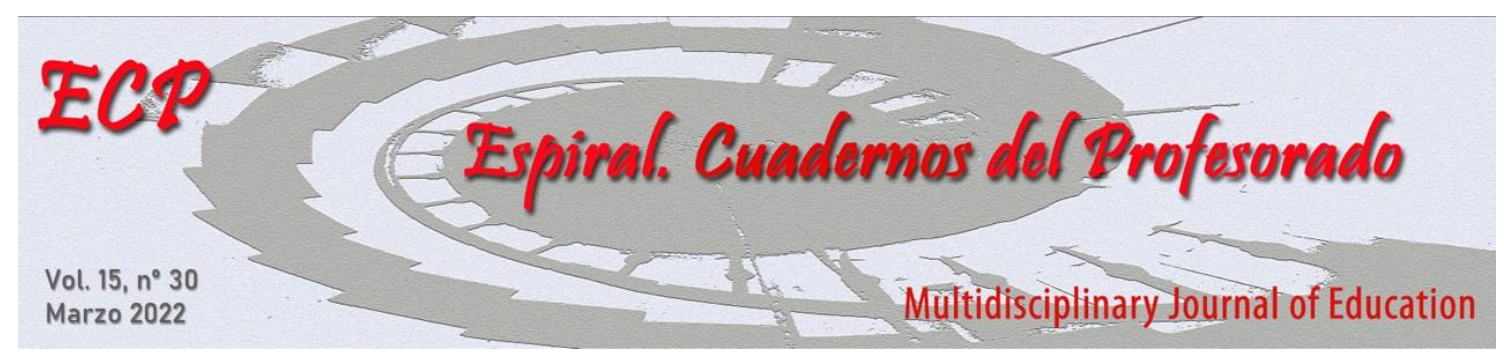

\title{
Desarrollo del currículo estructurado con base en proyectos formativos: Experiencia universitaria durante confinamiento social
}

\section{Development of the structured curriculum based on training projects: University experience during social confinement}

\author{
Edwin Roger Esteban Rivera, Amancio Ricardo Rojas Cotrina, Sonia Fiorella Callupe \\ Becerra, y Jorge Boyle Chávez Albornoz
}

Universidad Nacional Hermilio Valdizán, Perú

\begin{abstract}
Resumen
Debido a la propagación de la COVID-19 en el mundo, casi la totalidad de países cerraron sus fronteras y decretaron confinamiento social, "obligando" a que la educación formal presencial migre hacia la educación a distancia. Las instituciones educativas se fueron adecuando a la educación a distancia acorde a sus posibilidades, dejando al descubierto las enormes brechas digitales que existen entre los estudiantes, instituciones educativas, ciudades y clases sociales. En estas condiciones, el programa Ciencias Históricos Sociales y Geográficas de la Universidad Nacional Hermilio Valdizán, Perú, inició el proceso de ejecución de un currículo transdisciplinar, estructurado con base en proyectos formativos que, por su naturaleza, requiere del contacto directo entre estudiantes y el entorno social para resolver problemas del contexto, como un medio para desarrollar las competencias previstas. La investigación, que tiene como objetivo describir la ejecución de los proyectos formativos en la modalidad online e interpretar las experiencias de los estudiantes, se realizó con el método fenomenológico-hermenéutico que permitió establecer seis categorías de los relatos de las experiencias de los estudiantes: Nueva experiencia formativa, promoción de la lectura, divergencia de información, trabajo colaborativo, transdisciplinariedad y articulación aprendizaje-investigación; evidenciando que es posible desarrollar currículo transdisciplinar en tiempos de confinamiento social.
\end{abstract}

Palabras clave: Competencias; socioformación; currículo transdisciplinar; confinamiento social; universidad.

\begin{abstract}
Due to the spread of COVID-19 in the world, almost all countries closed their borders and decreed social confinement, "forcing" face-to-face formal education to migrate to distance education. Educational institutions gradually adapted to distance education according to their possibilities, exposing the enormous digital gaps that exist between students, educational institutions, cities and social classes. Under these conditions, the Social History and Geographical Sciences program of the Hermilio Valdizán National University, Peru, began the process of executing a transdisciplinary curriculum, structured based on training projects that, by its nature, requires direct contact between students and the social environment to solve context problems, as a means to develop the expected competencies. The research, which aims to describe the execution of the training projects in the online mode and interpret the experiences of the students, was carried out with the phenomenological-hermeneutical method that allowed establishing six categories of the accounts of the students' experiences: New formative experience, reading promotion, information divergence, collaborative work, transdisciplinarity and learning-research articulation; evidencing that it is possible to develop transdisciplinary curriculum in times of social confinement.
\end{abstract}

Keywords: Competences; socioformation; transdisciplinary curriculum; social lockdown; University. 
Fecha de recepción: 20/06/2021

Fecha de aceptación: 13/09/2021

Correspondencia: Edwin Roger Esteban Rivera, Universidad Nacional Hermilio Valdizán, Perú Email: edroer@gmail.com

\section{Introducción}

El mundo actual requiere profesionales que resuelvan problemas en diversas situaciones y contextos y, sobre todo, que actúen en el marco de un sólido proyecto ético de vida. La tarea de la universidad es formar profesionales con el perfil que demanda la sociedad. Formar profesionales acorde a las exigencias actuales implica diseñar modelos educativos y currículos universitarios orientados al desarrollo de competencias (Zapata, 2015; González \& Acevedo, 2016; Guzmán, 2017; Piñero et al., 2021).

Bajo estas exigencias, el modelo educativo de la Universidad Nacional Hermilio Valdizán (UNHEVAL) de Huánuco, Perú, "busca formar personas con un sólido proyecto ético para mejorar las condiciones de vida en la comunidad, generar sustentabilidad ambiental y contribuir a lograr la sociedad del conocimiento en el marco del trabajo colaborativo y el abordaje de problemas" (UNHEVAL, 2017, p. 87). El modelo se sustenta en el enfoque socioformativo que, prioriza la formación de personas emprendedoras y éticas, capaces de resolver problemas del contexto, con pensamiento crítico y sistémico, gestión y co-creación del conocimiento, la metacognición y la aplicación de la investigación formativa y científica, mediante proyectos colaborativos a fin de mejorar las condiciones de vida, con respeto a la diversidad sociocultural (Tobón, 2015; Tobón et al., 2016; Esteban et al., 2021).

Asumir nuevas perspectivas educativas implica cambiar la praxis educativa. En la UNHEVAL significó pasar de un currículo por objetivos a un currículo basado en competencias (Esteban et al., 2021). Sobre el diseño de currículos basado en competencias existen varias propuestas, entre ellas destaca el funcionalista, conductual-organizacional, constructivista y socioformativo. El primero conceptúa a la competencia como desempeño de funciones laborales; el segundo como actuación con base en conductas que aportan ventajas competitivas a las organizaciones; el tercero, como el desempeño en procesos laborales y sociales dinámicos, abordando las disfunciones que se presenta; y el cuarto, como actuaciones integrales ante problemas y situaciones de la vida con idoneidad ética y mejora continua (Tobón, 2015; Martínez et al., 2019).

$\mathrm{Al}$ concebir que las competencias son actuaciones integrales para resolver problemas del contexto con metacognición, se asume que los currículos deben formar integralmente a los estudiantes, articulando el saber ser, saber conocer, saber hacer y saber convivir; en el marco de la inter y transdisciplinariedad (Tobón, 2015; Guzmán, 2017); vale decir, estructurar el currículo articulando disciplinas o campos del saber, siendo una opción los proyectos formativos (Tobón, 2013; Zapata, 2015; Tobón et al., 2015; Guzmán, 2017). La articulación de diversas disciplinas, permite interpretar la realidad y los fenómenos de manera holística (Trujillo et al., 2020; Bennásar, 2020).

Considerando que el currículo es "la concreción especifica de una teoría pedagógica para volverla efectiva y asegurar el aprendizaje y desarrollo de un grupo particular de alumnos para la cultura, época y comunidad de la que hacen parte" (Flores, 2003, p. XXVI), la UNHEVAL optó por la socioformación que, es "un enfoque de gestión del talento humano que busca formar personas para vivir en la sociedad del conocimiento, con un proyecto ético de vida sólido y con las competencias necesarias para contribuir a resolver problemas que se presentan en los diferentes contextos" (Tobón, 2014, p. 27), buscando permanentemente una mejor calidad de vida, tanto en el plano personal como social (García et al., 2014). En la socioformación, el proyecto ético de vida es concebido como el proceso mediante el cual "cada persona planea, vive $\mathrm{y}$ valora su vida con base en metas que responden a sus necesidades vitales de crecimiento y

Espiral. Cuadernos del Profesorado | ISSN 1988-7701 | 2022, 15(30), 24-37 
Desarrollo del currículo estructurado con base en proyectos formativos: Experiencia universitaria durante confinamiento social

desarrollo, acorde con la construcción, reflexión, apropiación y aplicación de valores universales" (Tobón, 2013, p, 118).

La socioformación propugna la inter y transdisciplinariedad, por eso enfatiza en la estructuración curricular con base en proyectos formativos en reemplazo de las asignaturas, "consiste en un conjunto articulado de actividades de aprendizaje, enseñanza y evaluación para resolver un determinado problema del contexto y contribuir al desarrollo de una competencia, a través de la elaboración de uno o varios productos" (UNHEVAL, 2017, p. 87). Este enfoque plantea que para desarrollar competencias se "requiere de nuevos ambientes de formación que trasciendan el énfasis en contenidos, la linealidad del aprendizaje, la fragmentación de las asignaturas y se centren en problemas contextualizados a partir de la colaboración" (Parra et al., 2015). Un proyecto formativo debe ser abordado de manera holística, traspasando los límites de las asignaturas. No se trata de fraccionar un proyecto en segmentos donde cada docente "se hace cargo" de una parte, se trata que los docentes actúen colaborativamente, en equipo, con la mirada puesta en que los estudiantes y docentes aporten a la solución del problema del contexto real identificado.

Con estas prerrogativas teóricas, el programa académico de Ciencias Histórico Sociales y Geográficas, Facultad Ciencias de la Educación, diseñó el currículo con base en proyectos formativos que, en concordancia a la Ley Universitaria 30220 (Congreso de la República, 2014), la formación profesional se realiza en cinco años, equivalente a diez ciclos académicos. El plan de estudios consta de 30 proyectos formativos, en cada proyecto formativo se aborda un problema del contexto. En el I y II ciclo se desarrollan cuatro proyectos formativos en cada ciclo, en el III, IV, V, VI, VI y VII tres; y en el IX y X dos proyectos formativos.

La entrada en vigencia del currículo con base en proyectos formativos el año 2020, coincidió con la implementación de políticas de aislamiento social obligatorio a escala global por la amenaza de la COVID-19, restringiéndose las clases presenciales y masificándose la educación a distancia (Esteban et al., 2020; García \& Taberna, 2020; Martínez, 2020; Esteban et al., 2021; Piñero et al., 2021).

Uno de los proyectos formativos contemplado en el III ciclo es Aplicando las nuevas tendencias didácticas, que se desarrolló en 16 semanas, acumulando 192 horas y 8 créditos académicos. Debido a la coyuntura sanitaria se asumió el reto de desarrollar aprendizajes pertinentes en condiciones de aislamiento social obligatorio aplicando nuevas tendencias didácticas. El artículo tiene como propósito interpretar las experiencias de los estudiantes en el desarrollo del proyecto formativo en la modalidad online.

\section{Método}

\section{Enfoque metodológico}

La investigación se sustenta en el paradigma interpretativo, dado que este paradigma concibe a la realidad social y cultural pasible de ser interpretada (Sandín, 2003; Rivas, 2014). Se asumió la perspectiva metodológica cualitativa, específicamente el método fenomenológicohermenéutico que "conduce a la descripción e interpretación de la esencia de las experiencias vividas, reconoce el significado y la importancia en la pedagogía, psicología y sociología según la experiencia recogida" (Fuster, 2019). Este método enfatiza en el marco de referencia interno del sujeto que vive y experimenta un determinado proceso, persigue la descripción e interpretación de significados de vida, de esencias vividas, de aspectos existenciales del sujeto y asimismo, "ve" el mundo, la realidad, el fenómeno en estudio o al propio sujeto desde sus puntos de vista (Sandín, 2003; 2009; Piñero et al., 2019). En nuestro caso se empleó para interpretar y comprender la experiencia de los estudiantes universitarios en el proceso de ejecución del currículo basado en proyectos en la modalidad online, debido al confinamiento social obligatorio por COVID-19. 


\section{Diseño}

En la perspectiva de investigación cualitativa, el diseño es una fase preparatoria de la investigación, es ahí donde se planifican las actuaciones y procedimientos de la investigación, es flexible y marca la ruta de trabajo del investigador cualitativo (Ayala, 2008; Mardones et al., 2018; Piñero et al., 2019; Fuster, 2019). El diseño de la presente investigación consta de cuatro fases: Planificación de la investigación, recogida de información de la experiencia vivida, reflexión de acerca de la experiencia vivida y expositiva teórica, las mismas que se especifican en la Tabla 1.

Tabla 1.

Fases y actividades del diseño metodológico.

\begin{tabular}{|c|c|}
\hline Fase & Actividades \\
\hline $\begin{array}{l}\text { Planificación de la } \\
\text { investigación }\end{array}$ & $\begin{array}{l}\text { 1. Clarificación de supuestos ante el tema de investigación. } \\
\text { 2. Definición de las preguntas orientadoras o guías iniciales. } \\
\text { 3. Análisis de las teorías que orientan la investigación. } \\
\text { 4. Elección de las técnicas e instrumentos. }\end{array}$ \\
\hline $\begin{array}{lc}\text { Recogida } & \text { de } \\
\text { información de } & \text { la } \\
\text { experiencia vivida } & \end{array}$ & $\begin{array}{l}\text { 1. Registro de las sesiones de aprendizaje en los diarios de campo. } \\
\text { 2. Entrevistas en profundidad a estudiantes que experimentaron el proceso. }\end{array}$ \\
\hline $\begin{array}{l}\text { Reflexión de acerca } \\
\text { de la experiencia } \\
\text { vivida }\end{array}$ & $\begin{array}{l}\text { 1. Categorización de los diarios de campo y transcripciones de las entrevistas. } \\
\text { 2. Identificación de los temas experienciales. } \\
\text { 3. Comprensión de la experiencia: elaboración de significados de un mismo } \\
\text { hecho. }\end{array}$ \\
\hline Expositiva teórica & $\begin{array}{l}\text { 1. Integración de las estructuras particulares en una estructura general. } \\
\text { 2. Confrontación de los hallazgos con otros estudios. } \\
\text { 3. Configuración y presentación del informe final. }\end{array}$ \\
\hline
\end{tabular}

Nota. Elaboración propia.

\section{Desarrollo del proyecto formativo}

El proyecto formativo Aplicando las nuevas tendencias didácticas se realizó en seis fases (Tobón, 2014; Tobón et al., 2015).

\section{Fase 1: Presentación del proyecto}

El primer día de clases se socializó con los estudiantes el proyecto formativo que comprende: Información general, propósito, competencias, problema del contexto, saberes esenciales, programa de actividades, matriz de valoración de desempeños y referencias. Los estudiantes constituyeron seis equipos de trabajo, cada equipo identificó un problema vinculado al desarrollo de clases en tiempos de confinamiento social, las disciplinas que contribuyen a la solución del problema y las acciones claves. También se establecieron las evidencias a entregar durante el desarrollo del proyecto.

\section{Fase 2: Valoración de los saberes previos}

Mediante la lluvia de ideas se identificaron los conocimientos, saberes e información del alumnado con relación a los aspectos básicos vinculados al proyecto, tales como: COVID-19 y aislamiento social, currículo nacional, competencias, corrientes pedagógicas, estrategias didácticas, nuevas tendencias didácticas, educación virtual, neurociencia del aprendizaje, dominio de las tecnologías de la información y comunicación (TIC), manejo de fuentes de información y redacción de informes, los mismos que fueron tomados en cuenta en el desarrollo de los proyectos formativos. 
Desarrollo del currículo estructurado con base en proyectos formativos: Experiencia universitaria durante confinamiento social

\section{Fase 3: Gestión del conocimiento}

Los estudiantes exploraron diversas fuentes de información y seleccionaron las pertinentes, analizaron, recrearon y sistematizaron la información para los lograr los objetivos planteados por cada equipo.

\section{Fase 4: Diagnóstico del contexto}

Los equipos libremente determinaron con qué estudiantes de la educación básica regular trabajar y abordar el reto: Aplicando las nuevas tendencias didácticas en condiciones de confinamiento social obligatorio por el COVID-19, diagnosticaron sus necesidades de aprendizaje, las condiciones de conectividad y los equipos con que cuentan los estudiantes.

\section{Fase 5: Emprendimiento y resolución del problema}

Sustentado en teorías vigentes, como el conectivismo desarrollado por George Siemens y por Stephen Downes (Siemens, 2010; Gallego, 2017; Cueva et al., 2019; Torres \& Barnabé, 2020), clases motivantes y participativas (Tobón, 2019), formación de investigadores en la universidad (Stenhouse, 2007; Juárez et al., 2019), cada equipo diseñó estrategias para promover aprendizajes pertinentes en condiciones de aislamiento social obligatorio y aplicó diversos recursos de comunicación acordes a la realidad de los estudiantes de educación básica, toda vez que la socioformación promueve la realización de proyectos de emprendimiento que tengan impacto en la resolución de problemas individuales y colectivos que se tienen en la cotidianidad (Juárez et al., 2019).

\section{Fase 6: Socialización del desarrollo del proyecto formativo}

La evidencia final consistió en la presentación del artículo completo por cada equipo de trabajo. Ver tabla 2.

Tabla 2.

Artículos presentados por los equipos de trabajo.

\begin{tabular}{cl}
\hline $\begin{array}{c}\text { Equipo } \\
\mathbf{N}^{\circ}\end{array}$ & \multicolumn{1}{c}{ Título del artículo } \\
\hline 1 & Educación virtual en tiempos de COVID-19 \\
2 & Aprendizaje de los estudiantes del nivel secundario, a través de medios virtuales \\
3 & Estrategias didácticas para la educación virtual en contexto del aislamiento social obligatorio \\
4 & La educación a distancia y el proceso de aprendizaje en tiempos del COVID-19 \\
5 & Las herramientas tecnológicas para el desarrollo de las clases virtuales en tiempos de COVID-19 \\
6 & Desarrollo de clases a través del aplicativo WhatsApp \\
\hline
\end{tabular}

Nota. Elaboración propia.

El artículo se presentó con los siguientes elementos: título, resumen, introducción, fundamentación teórica, metodología, resultados, conclusiones, referencias y anexos. Este producto es coherente con el modelo educativo de la universidad y el currículo de la carrera profesional que, enfatizan en la articulación de las actividades lectivas con la investigación, a fin de familiar a los estudiantes con la investigación y difusión de la misma, toda vez que una modalidad de titulación es la sustentación de un artículo científico publicado en una revista indexada.

\section{Participantes}

En el proyecto se inscribieron 28 estudiantes, 15 de ellos varones y 13 mujeres; 4 dejaron de estudiar por motivos de conectividad a internet: 3 varones y 1 mujer. Se trabajó con 24 estudiantes (12 varones y 12 mujeres) que participaron con regularidad en las clases virtuales del proyecto Aplicando las nuevas tendencias didácticas, semestre académico 2020-I, de la Escuela 
Profesional Ciencias Histórico Sociales y Geográficas, perteneciente a la Facultad Ciencias de la Educación, UNHEVAL.

\section{Instrumentos}

La recogida de información se realizó mediante la guía de revisión documental, diarios de campo y guías de entrevista en profundidad a estudiantes de pregrado. Mediante la guía revisión documental se analizaron libros especializados en currículos por competencias y socioformación; y artículos publicados en revistas indizadas que abordan sobre currículo por competencias y ejecución curricular en la modalidad a distancia. Se priorizaron artículos de los últimos cinco últimos años.

Las 30 sesiones virtuales que comprendió el desarrollo del proyecto formativo fueron grabadas en el aplicativo de videoconferencia Cisco Webex, formato ARF. Después de cada clase, las grabaciones fueron transcritas en los diarios de campo para posteriormente ser categorizadas.

La guía de entrevista estuvo centrada en recoger información sobre las experiencias vividas por los estudiantes en el desarrollo del proyecto formativo Aplicando las nuevas tendencias didácticas en periodo de confinamiento social obligatorio.

\section{Procedimiento}

En la investigación fenomenológica hermenéutica la entrevista en profundidad permite recoger información sobre la experiencia de quienes vivieron un fenómeno en particular, en este caso de quienes participaron en el proyecto formativo en condiciones de confinamiento social. Por consiguiente, de los 24 estudiantes de las clases se seleccionaron a 15 estudiantes para la entrevista en profundidad, por medio del muestreo propositivo. Se tuvo en consideración el conocimiento del tema (Mendieta, 2015) y la predisposición de para formar parte de los informantes.

Las entrevistas en profundidad se realizaron de manera virtual, se empleó el aplicativo de videoconferencia Cisco Webex; por consiguiente, las entrevistas quedaron grabadas en el sitio del aplicativo. Las entrevistas fueron transcritas en el formato de protocolo de registro de la información para luego ser categorizadas.

Para garantizar la calidad de la información se crearon condiciones favorables o rapport para que los entrevistados respondan con libertad y confianza a las preguntas, también se transcribieron las experiencias tal y como narraron los entrevistados. Además, se realizó la triangulación que, consiste "en determinar ciertas interacciones o coincidencias a partir de diferentes apreciaciones y fuentes informativas o varios puntos de vista del mismo fenómeno" (Leal, 2009, p. 135), las mismas que fueron enriquecidas con la corroboración teórica de libros y artículos de revistas.

\section{Análisis de datos}

La sistematización y análisis de la evidencia se realizó mediante la categorización. La categorización hace referencia a "los procedimientos empleados en la investigación cualitativa para transformar la evidencia y dar sentido y significado a la realidad expresada por el informante, y que el investigador vincula mediante una frase, una palabra o concepto" (Piñero et al., 2019, p. 201). En el proceso de categorización se cumplieron las tres etapas: codificación abierta, codificación axial y codificación selectiva (Esteban et al., 2018).

"La codificación abierta es el proceso analítico por medio del cual se identifican los conceptos y se descubren en los datos sus propiedades y dimensiones" (Strauss \& Corbin, 2012, p. 110). La codificación abierta es insumo para la codificación axial, que es concebida como "el proceso de relacionar las categorías a sus subcategorías, denominado "axial", porque la codificación ocurre alrededor del eje de una categoría, y enlaza las categorías en cuanto a sus propiedades y dimensiones" (Strauss \& Corbin, 2012, p. 134). Finalmente se procedió a la codificación selectiva que, es el "proceso de integrar y refinar la teoría (Strauss \& Corbin, 2012, p. 157), profundizando "en las conceptualizaciones alrededor de una categoría central, 
Desarrollo del currículo estructurado con base en proyectos formativos: Experiencia universitaria durante confinamiento social

recopilando nuevos datos de ser necesario, con el propósito de lograr mayor definición de la categoría" (Monge, 2015, p. 80). Estos procesos se realizaron sin emplear ningún software, lográndose categorías emergentes de las entrevistas en profundidad a los estudiantes de pregrado respecto a sus experiencias y vivencias en la implementación del currículo transdisciplinar basado en proyectos formativos.

El proceso seguido fue el siguiente: Se realizó la transcripción de las entrevistas en el formato de protocolo de registro de la información que, consta de tres columnas: $\mathrm{N}^{\circ}$ de línea, textos y categorías o unidades de significación. Al interpretar el texto se asignaron categorías o unidades de significación en la respectiva columna, para un manejo didáctico se empleó la cromatización. Se integraron o agruparon categorías en una más amplia y comprensiva, estableciendo nexos. Se mantuvo permanente comparación hasta alcanzar la saturación de las categorías; es decir, hasta cuando de las entrevistas ya no emergían "propiedades, dimensiones, o relaciones nuevas durante el análisis" (Strauss \& Corbin, 2012, p. 134). Al analizar diez entrevistas, no se notó el surgimiento de nuevas informaciones, razón por la cual se consideró que la información recogida era suficiente con relación al objetivo de la investigación.

\section{Categorías de Análisis}

Las categorías que emergieron de la observación y la entrevista en profundidad a estudiantes de pregrado se describen en la tabla 3.

Tabla 3.

Categorías de análisis.

\begin{tabular}{|c|c|}
\hline Categoría & Descripción \\
\hline $\begin{array}{l}\text { Nueva experiencia } \\
\text { formativa }\end{array}$ & $\begin{array}{l}\text { Concebida como las condiciones en que se realiza el proceso de enseñanza } \\
\text { y aprendizaje. }\end{array}$ \\
\hline Creatividad & Concebida como las. \\
\hline Promoción de la lectura & $\begin{array}{l}\text { Concebida como el fomento a la lectura en el desarrollo de los proyectos } \\
\text { formativos. }\end{array}$ \\
\hline $\begin{array}{l}\text { Divergencia de } \\
\text { información }\end{array}$ & $\begin{array}{l}\text { Concebida como las contradicciones o diferentes puntos de vista que tienen } \\
\text { los especialistas sobre un tema en particular. }\end{array}$ \\
\hline Trabajo colaborativo & $\begin{array}{l}\text { Concebida como la realización de una actividad mediante la articulación de } \\
\text { acciones, fortalezas y recursos de varias personas, a partir del acuerdo de un } \\
\text { plan de acción. }\end{array}$ \\
\hline Transdisciplinariedad & $\begin{array}{l}\text { Concebida como la articulación de las áreas o disciplinas para abordar un } \\
\text { problema o caso. }\end{array}$ \\
\hline $\begin{array}{l}\text { Articulación aprendizaje - } \\
\text { investigación }\end{array}$ & $\begin{array}{l}\text { Concebida como la integración de la investigación en el desarrollo de los } \\
\text { proyectos formativos. }\end{array}$ \\
\hline Aprendizaje autónomo & $\begin{array}{l}\text { Concebida como la capacidad de aprender por uno mismo, estableciendo } \\
\text { objetivos y procedimientos. }\end{array}$ \\
\hline
\end{tabular}

Nota. Elaboración propia. 


\section{Resultados}

\section{Nueva experiencia formativa}

En Perú muchas universidades diseñaron sus currículos basado en competencias, pero ninguna estructuró el currículo transdisciplinar con base en proyectos formativos. Por tanto, esta experiencia fue novedosa para los estudiantes, los mismos que refieren:

"Me chocó bastante trabajar los proyectos, yo vengo de una generación de estudiantes que tuvo una educación tradicional, acostumbrada a trabajar con asignaturas y no con proyectos" (E-E1).

"Fue una experiencia nueva, tanto para nosotros como para los docentes, si bien, nadie tenía pensado trabajar de esta manera, poco a poco nos fuimos adaptando a trabajar virtualmente y sobre todo a trabajar por proyectos" (E-E17).

"El proyecto fue fascinante (...), veo gran diferencia entre cursos y proyectos, porque los cursos son más teóricos, los proyectos son teóricos y prácticos, sobre todo te permite resolver problemas" (E-E3).

"Nos pudimos adaptar a este nuevo contexto que no es fácil, por ejemplo, hay muchas universidades que he escuchado que no se pudieron adaptar, en nuestro caso lo hicimos bien y estamos teniendo resultados" (E-E12).

"Cambiar de currículo en este momento que estamos pasando por el COVID-19, ha sido un reto para la carrera, nos costó inicialmente, pero vale la pena. Los proyectos nos enseñan a realizarnos como persona" (E-E10).

Las narraciones muestran que no es fácil dejar de hacer lo habitual. Todo cambio cuesta, pero que poco a poco uno se va acostumbrando a lo nuevo y en algún momento formará parte de lo habitual. Los estudiantes interiorizaron que la esencia de los proyectos formativos es la resolución de problemas del contexto (Tobón, 2014; Parra et al., 2015). Se entiende por problema del contexto a una situación adversa que aqueja el entorno social en el que se desenvuelven los estudiantes, frente a ella se asume el reto de contribuir a su transformación en una situación ideal o esperada (UNHEVAL, 2017).

\section{Creatividad}

Los equipos realizaron sesiones de aprendizaje haciendo uso de diversos medios, algunos emplearon plataformas virtuales y otros aplicativos de videoconferencia como el Zoom, Jitsi Meet, BigBlueButton, WhatsApp. Cuando los estudiantes no contaban con acceso a ninguno de estos aplicativos, hicieron uso de llamadas telefónicas. En todo el proceso se promovió la creatividad.

$\mathrm{Al}$ respecto los estudiantes manifiestan:

"El trabajo por proyectos promueve nuestra creatividad porque tenemos [que] idear posibilidades y estrategias para resolver el problema que hemos elegido" (E-E7).

"Se promueve la originalidad de los proyectos, hay un sentido más de competitividad, de exigencia, eso es bueno para la carrera. Cuando hay exigencia es fácil adaptarse" (EE2).

\section{Promoción de la lectura}

Esta fue una de las categorías de mayor saturación, los estudiantes manifiestan:

"El trabajo por proyectos formativos me dejó un gran aprendizaje, porque te exige a leer muchos documentos y a través de eso mejoras tu comunicación” (E-E1).

"Cuanto más lees, quieres saber más, cuando estás leyendo encuentras muchas cosas, tienes más vocabulario y se llega a una investigación más profunda. En las asignaturas no encuentras eso" (E-E2). 
Desarrollo del currículo estructurado con base en proyectos formativos: Experiencia universitaria durante confinamiento social

"Los proyectos nos hicieron dar cuenta que debemos seguir leyendo más, en estas vacaciones leeremos más artículos para fortalecer nuestro aprendizaje. Me gustaría seguir con los proyectos, porque en las asignaturas no había tanto esfuerzo como se requiere ahora" (E-E4).

Los proyectos formativos resuelven problemas del contexto (Parra et al., 2015), para resolverlos se requiere conocer el problema, leer sobre él, analizar las posibles soluciones; así mismo, para elaborar el informe es necesario conocer la estructura de los informes, las características de cada una de las partes del informe y sobre todo articular las ideas. Para todo este proceso se requiere leer, la lectura abre las puertas del conocimiento.

\section{Divergencia de Información}

Analizar textos permite al estudiante comprender la información, también a encontrar similitudes y discrepancias entre los planteamientos de los autores. Al respecto expresan los estudiantes:

"Al trabajar el proyecto, revisas artículos que se contradicen, entonces te lleva a comprobar si lo que dice un autor es verdad" (E-E9).

"Los proyectos nos llevan a investigar en fuentes confiables, inicialmente no nos dábamos cuenta que algunas fuentes no son tan confiables, ahora se buscar información en fuentes confiables para desarrollar las actividades" (E-E17).

Las contradicciones impulsan el desarrollo del conocimiento, así asumieron los estudiantes. Ante contradicciones encontradas, investigaron para saber qué propuesta tiene mayor argumento o se aproxima más a la realidad de los estudiantes. Entendieron que existen muchas formas de hacer ciencia y que el conocimiento en las ciencias sociales no es convergente, sino divergente (Piñero, et. al., 2019).

\section{Trabajo Colaborativo}

A la mayoría de las personas les resulta más fácil trabajar solos, pero al comprender que se puede mejorar el trabajo con la ayuda de los demás, van interiorizando la necesidad de trabajar colaborativamente para optimizar los resultados. Los estudiantes narran:

"Aprendi a trabajar entre varias personas, el trabajo colaborativo mejor dicho, a adecuarme a la situación de la otra persona, también a entender y buscar soluciones para poder realizar los trabajos" (E-E9).

"Es muy importante llevar proyectos, nos ayudó a reforzar nuestras capacidades sociales, mediante reuniones de Zoom intercambiamos ideas y colaborábamos entre todos nosotros, como dicen dos cabezas piensan mejor que uno, entonces todos nos ayudábamos para sacar un buen producto" (E-E7).

"Desarrollamos el trabajo colaborativo en todo momento, un claro ejemplo es la elaboración del artículo, todos dimos aporte para las tablas estadísticas, todos estábamos metidos para que nuestro artículo quede bien presentable” (E-17).

El trabajo colaborativo es muy importante en los proyectos formativos porque permite a las personas lograr metas en común, mediante la complementación y articulación de sus competencias. El trabajo colaborativo implica las siguientes acciones: Acordar una meta común a lograr, trabajar en función a un plan de acción acordado entre todos, trabajar con sinergia, actuar con metacognición, interactuar con comunicación asertiva y actuar con responsabilidad personal (Tobón, 2017; Vázquez et al., 2017).

\section{Transdisciplinariedad}

La realidad está interrelacionada, por tanto, si se desea estudiar un fenómeno o resolver un problema, se debe enfocar de manera holística, no de manera fragmentada. El desarrollo curricular a través de los proyectos formativos propicia un abordaje inter y transdisciplinar, como se evidencia en la siguiente expresión: 
"Es muy interesante el propósito de los proyectos, te permiten profundizar en problemas actuales y darles una solución, juntando varias disciplinas" (E-E5).

"Con las asignaturas uno profundiza en un tema en particular, en una ciencia determinada. Cuando se trabaja por proyectos debes analizar un problema desde varias ciencias, plantear y ejecutar alternativas de solución amparados en varias disciplinas científicas o ciencias. A media que vas profundizando en el estudio, se hace necesario revisar otros planteamientos teóricos" (E-E12).

Efectivamente, la transdisciplinariedad se caracteriza por la articulación de diversos saberes, disciplinares y no disciplinares, se concibe que no existe un método sino que este se desarrolla según el problema, siendo necesario diseñar y hacer uso de herramientas para la interacción de los diversos actores (Moreno, 2017).

\section{Articulación Aprendizaje-investigación}

El requerimiento que se hace a la educación actual es la articulación del desarrollo curricular con la investigación. Stenhouse (2007) considera que no es posible separar la enseñanza de la investigación. Los proyectos formativos refuerzan esta postura porque:

"Cuando se trabaja los proyectos formativos, todo es investigación, aprendemos investigación haciendo investigaciones, también aprendimos a elaborar artículos" (GFE15).

"Me resultaba dificil al inicio desarrollar el proyecto porque todo es investigación, tenemos que identificar un problema, estudiarla desde distintas bases teóricas, debemos formular objetivos y plantear alternativas para solucionar el problema. Al final redactamos un artículo como evidencia de nuestro proyecto, que considero es un verdadero trabajo de investigación" (EP-E2).

En la tarea de formar investigadores, los proyectos formativos se constituyen en una opción, al tiempo de ir desarrollando el pensamiento complejo en los estudiantes que, según el modelo educativo de la universidad es concebido como "el análisis y resolución de un problema desde diferentes ángulos, mediante la articulación de saberes y perspectivas de varias áreas y campos, con análisis crítico, creatividad, flexibilidad, y gestión y co-creación del conocimiento" (UNHEVAL, 2017, p. 86).

\section{Aprendizaje Autónomo}

La sociedad demanda que la universidad y la educación en general formen estudiantes competentes, que construyan sus aprendizajes de manera autónoma. Los proyectos formativos es un medio para estos propósitos.

$\mathrm{Al}$ respecto, los estudiantes expresan:

"Al inicio no sabía qué hacer, nunca había hecho clases. Me ponía nervioso, poco a poco me fui preparando y, este, mis clases mejoraron" (E-E1).

"La primera vez me sobró tiempo, creo que no me preparé bien. Los consejos del profesor y al leer más, siento que ahora dicto mejor las clases" (E-E3).

"Después de mis clases pensaba qué hice mal y qué hice bien, asi pude mejorar" (EE12).

Los estudiantes de pregrado asumieron el "rol docente", se autoevaluaron permanentemente para mejorar su práctica, es decir, asumieron una investigación-acción pedagógica (Esteban et al., 2018). Al respecto expresan:

\section{Discusión y Conclusiones}

La virtualización de la educación es una de las tendencias más notables de las últimas décadas, tendencia que se hizo más evidente con el confinamiento social obligatorio por COVID19, decretado en la mayoría de los países del mundo. La educación formal presencial dio paso 
Desarrollo del currículo estructurado con base en proyectos formativos: Experiencia universitaria durante confinamiento social

"forzado" a una educación a distancia en todos los niveles educativos (Esteban et al., 2020; García \& Taberna, 2020), poniéndose en evidencia la brecha digital existente en el mundo. A la par que existen instituciones educativas cuyos estudiantes cuentan con computadores y acceso a internet, existen otras donde no existe señal de internet ni de telefonía. El escenario actual nos muestra que la inclusión digital en el ámbito educativo es un proceso incipiente, y que afecta directamente a colectivos en riesgo de exclusión social (Martínez, 2020), que optan por abandonar sus estudios.

En la Universidad Nacional Hermilio Valdizán de Huánuco, Perú, no solo se tuvo que afrontar el tránsito "forzado" a la educación virtual, sino también el reto de ejecutar un currículo transdisciplinar estructurado con base en proyectos formativos que, por su naturaleza, requiere el contacto directo de los estudiantes con el entorno para resolver problemas del contexto, todo ello orientado a que el estudiante desarrolle competencias en situaciones reales, que le permita afrontar las adversidades e incertidumbres de un mundo complejo y cambiante (García, et al., 2014; Guzmán, 2017; Juárez et al., 2019; Tobón et al., 2015; Vázquez et al., 2017).

La ejecución de los proyectos formativos en la modalidad online significó para los estudiantes una nueva y fascinante experiencia. Al inicio les costó adecuarse pero paulatinamente se adaptaron. El principal recurso de las clases online fue la videoconferencia (Herrera \& Toro, 2020) y permitió a los estudiantes desarrollor "la responsabilidad, autonomía y una opción flexible para el aprendizaje" (Esteban et al., 2020).

Sin embargo, esta nueva experiencia también significó para algunos estudiantes una frustración, puesto que no contaban con dispositivos electrónicos para acceder a internet o carecían de conectividad. Estudios realizados en la Pontificia Universidad Católica del Perú (Lovón \& Cisneros, 2020) y en la Universidad Nacional Hermilio Valdizán (Campos et al., 2020), coinciden en que la sobrecarga académica de las clases virtuales generó estrés en los estudiantes que contaban con adecuados recursos tecnológicos; en tanto, en estudiantes carentes de óptimos recursos tecnológicos, además del estrés, generó frustración y deserción universitaria.

La operativización del currículo con base en proyectos formativos, desde la mirada estudiantil, incentivó el trabajo colaborativo entre los estudiantes porque el reto a lograr implicó el trabajo de todos integrantes del equipo, promueve la lectura, articula el proceso enseñanza y aprendizaje con la investigación y, promueve la transdisciplinariedad. A decir de Ramírez \& Lopera (2019, la inter y la transdisciplinariedad son potentes alternativas para la integración del conocimiento e investigación, transcendiendo los límites disciplinares e impulsando la construcción de modelos explicativos holísticos que den cuenta de la complejidad de los objetos de estudio y que ayuden a transformar los espacios tradicionales educativos, en espacios de construcción de nuevos conocimientos emergentes.

La educación virtual, adecuadamente llevada, permite un conjunto de ventajas como: los estudiantes van a su propio ritmo de aprendizaje y adquieren información en el momento en que lo necesitan (las clases quedan grabadas), permite la combinación de materiales auditivos, visuales y audiovisuales; tiende a ser interactiva; es flexible, el estudiante puede conectarse a las clases desde el lugar en que se encuentra; pone a disposición de los alumnos un amplio volumen de información; facilita la autonomía del estudiante; puede desarrollarse de manera sincrónica y asincrónica; ahorra costos y desplazamiento (Cabero, 2006; Esteban, et al., 2020).

Frente al nuevo escenario educativo urgen acciones gubernamentales y en cada una de las instituciones educativas para reducir las brechas digitales y desarrollar competencias en quienes lo requieren. Es un desafío para las universidades promover la cultura digital en los docentes y estudiantes, promover docentes que integren lo tecnológico, cognitivo, relacional y pedagógico. Es necesario el rediseño de una educación presencial hacia una remota o semipresencial, promover aprendizajes basados en retos o solución de problemas que, implica la articulación de diversos campos del saber y trabajo colaborativo de profesores; es decir, apostar por la inter y transdisciplinariedad curricular.

Contribución de cada Autor: "conceptualización, E.R.E.R. y S.F.C.B; análisis, E.R.E.R. y A.R.R.C.; escritura del manuscrito, J.B.C.A.; escritura, revisión y edición, A.R.R.C y S.F.C.B.; supervisión, E.R.E.R. 
Financiación: Esta investigación no recibió ninguna financiación externa.

Agradecimiento: A los estudiantes del proyecto formativo: Claudio Huanca, Renzo Monago, Geanpier Rojas, Libetho Sebastián, Nicole Illatopa, Karlizflor Jesús, Eliani Bonifacio, Pedro Solano, Yoel Encarnación, Estrellita Sebastián, Rudi Alania, Flor Urbano, Eliel Donato, Diana Reyes, Isaac Reyes, Chabeli Alvarado, Aquila Quiroz, Henryck Fretel, Gianella Vela, Javier Santiago, Evelyn Ponciano, Carolina Sifuentes, Rosa Blas, Benjamín Inocente.

Conflicto de Intereses: Los autores declaran que no tienen conflicto de intereses.

\section{Referencias}

Álvarez Álvarez, C., \& García Prieto, F. J. (2021). Brecha digital y nuevas formas académicas en la escuela rural española. Educar, 57(2), 397-411. https://doi.org/10.5565/rev/educar.1250

Ayala, R. (2008). La metodología fenomenológico-hermenéutica de M. Van Manen en el campo de la investigación educativa. Posibilidades y primeras experiencias. Revista de Investigación Educativa, 26(2), 409-430. https://revistas.um.es/rie/article/view/94001

Bennásar, M. (2020). Perspectivas curriculares para la enseñanza y aprendizaje de la educación física aplicada a la lúdica. Metropolitan, 1(1), 29-43.

Campos Cornejo, L. L., Jaimes Campos, M. Á., Villavicencio Guardia, M. C., \& Esteban Rivera, E. R. (2020). Bienestar y afrontamiento psicológico de estudiantes universitarios en estado de confinamiento por COVID-19. Revista Inclusiones, 7(Num. Especial), 377-398. https://bit.ly/3ssQEBY

Cabero, J. (2006). Bases pedagógicas del e-learning. Universities and Knowledge Society Journal, 3(1), 110. http://dx.doi.org/10.7238/rusc.v3i1.265

Congreso de la República. (2014). Ley Universitaria 30220. Diario oficial el Peruano: Normas Legales, XXXI(12914), 527211-527233. https://bit.ly/3Jbl1CJ

Cossío, M., Quiñones, O., \& Cossio, A. (2020). La continuidad pedagógica en educación superior en tiempos de la pandemia. Revista Publicando, 8(28), 67-73. https://doi.org/10.51528/rp.vol8.id2159

Cueva Delgado, J. L., García Chávez, A., \& Martínez Molina, O. A. (2019). El conectivismo y las TIC: Un paradigma que impacta el proceso. Revista Scientific, 4(14), 205-227. https://doi.org/10.29394/Scientific.issn.2542-2987.2019.4.14.10.205-227

Esteban, E., Cámara, A., \& Villavicencio, M. (2020). La educación virtual de posgrado en tiempos de COVID-19. Revista de Estilos de Aprendizaje, 13(Especial), 82-94. http://revistaestilosdeaprendizaje.com/article/view/2241

Esteban, E., Cámara, A., Rojas, A., \& Chávez, J. (2018). Investigación-acción pedagógica: Transformación de la práctica docente. UNHEVAL.

Esteban, E., Portocarrero, E., Rojas, A., Piñero, M., \& Callupe, S. (2021). La transdisciplinariedad desde el modelo educativo: una experiencia universitaria. Revista Inclusiones, 8(1), 241-261. http://revistainclusiones.org/index.php/inclu/article/view/197

Flores, R. (2003). Evaluación pedagogía y cognición. Mc Graw-Hill.

Fuster Guillen, D. E. (2019). Investigación cualitativa: Método fenomenológico. Propósitos y Representaciones, 7(1), 201-229. http://dx.doi.org/10.20511/pyr2019.v7n1.267

Gallego Torres, R. A. (2017). Antecedentes para el diseño de una nueva estrategia didáctica y de comunicación para el e-learning. Razón y Palabra, 21(3_98), 51-65. https://revistarazonypalabra.org/index.php/ryp/article/view/1042

García, J. A., López, N. M., \& Zúñiga, R. (2014). Aprendizaje y vida. Construccion didactica evaluacion y certificacion de competencias en educacion desde el enfoque socioformativo. Pearson.

García, M. I., \& Taberna, J. (2020). Transición de la docencia presencial a la no presencial en la UPC durante la pandemia del COVID-19. IJERI, International Journal of Educational Research and Innovation (15), 177-187. https://doi.org/10.46661/ijeri.5015

González, A., \& Acevedo, C. (2016). Percepción de estudiantes de la Salud acerca de la implementación de las macrocompetencias genéricas. Educación Médica Superior, 30(4), 349-360. 
Desarrollo del currículo estructurado con base en proyectos formativos: Experiencia universitaria durante confinamiento social

Guzmán, F. (2017). Problemática general de la educación por competencias. Revista Iberoamericana de Educación, 74, 107-120. https://rieoei.org/historico/documentos/rie74a04.pdf

Herrera Añazco, P., \& Toro Huamanchumo, C. (2020). Educación médica durante la pandemia del COVID -19: iniciativas mundiales para el pregrado, internado y el residentado médico. Acta Médica Peruana, 37(2), 169-175. https://doi.org/10.35663/amp.2020.372.999

Juárez, L. , Luna, J., \& Guzmán, C. (2019). Talento, investigación y socioformación. Kresearch.

Leal, J. (2009). La autonomía del sujeto investigador y la metodología de investigación (2 ${ }^{\mathrm{a}}$ ed.). Universidad de Los Andes.

Lovón Cueva, M. A., \& Cisneros Terrones, S. A. (2020). Repercusiones de las clases virtuales en los estudiantes universitarios en el contexto de la cuarentena por COVID-19: El caso de la PUCP. Propósitos y Representaciones, 8(SPE3), e588. http://dx.doi.org/10.20511/pyr2020.v8nSPE3.588

Mardones, R. E., Ulloa, J. B., \& Salas, G. (2018). Usos del diseño metodológico cualitativo en artículos de acceso abierto de alto impacto en ciencias sociales. FQS, Forum: Qualitative Social Research, 19(1), 1-18. de https://www.qualitative-research.net/index.php/fqs/article/view/2656/4168

Martínez, J. E., Tobón, S., \& López, E. (2019). Currículo: un análisis desde un enfoque socioformativo. Revista de Investigación Educativa de la REDIECH, 10(18), 43-63. https://dx.doi.org/10.33010/ie_rie_rediech.v10i18.200

Martínez, O. (2020). Brecha digital educativa. Cuando el territorio es importante. Sociedad e Infancias, 4, 267-270. https://doi.org/10.5209/soci.69629

Mendieta Izquierdo, G. (2015). Informantes y muestreo en investigación cualitativa. Investigaciones Andina, 17(30), 1148-1150. https://doi.org/10.33132/01248146.65

Monge, V. (2015). La codificación en el método de investigación de la grounded theory o teoría $\begin{array}{llll}\text { fundamentada. } & \text { Innovaciones } & \text { educativas, } & \text { 77(22), }\end{array}$ doi:https://doi.org/10.22458/ie.v17i22.1100

Moreno, L. A. (2017). Abordar lo complejo desde el diseño: Una mirada hacia la transdisciplinariedad. Revista Educación y Humanismo, 19(33), 369-385. https://doi.org/10.17081/eduhum.19.33.2650

Parra, H., Tobón, S., \& López, J. (2015). Docencia socioformativa y desempeño académico en la educación superior. Revista Paradigma, XXXVI(1), 42-55. http://ve.scielo.org/pdf/pdg/v36n1/art04.pdf

Piñero, M. L., Esteban, E. R., Rojas, A. R., \& Callupe, S. F. (2021). Tendencias y desafíos de los programas de posgrado latinoamericanos en contextos de COVID-19. Revista Venezolana de Gerencia, 26(93), 123-138. https://produccioncientificaluz.org/index.php/rvg/article/view/34972

Piñero, M. L., Rivera, M. E., \& Esteban, E. R. (2019). Proceder del investigador cualitativo: Precisiones para el proceso de investigación. Fabriray.

Ponce, I., Juárez, L. G., \& Tobón, S. (2020). Preferencias construcción y validación de un instrumento para evaluar el abordaje de la sociedad del conocimiento en docentes. Apuntes Universitarios. Revista $\begin{array}{lll}\text { de Investigación, } & \text { 40-65. }\end{array}$ https://apuntesuniversitarios.upeu.edu.pe/index.php/revapuntes/article/view/417/490

Ramírez, D., \& Lopera, C. (2019). Integración curricular basada en situaciones ambientales contextualizadas desde un enfoque transdisciplinar. Estado del arte. LAJSE. Revista Latin American Journal of Science Education, 6(2), 1-17. http://lajse.org/nov19/2019_22015_2.pdf

Rivas Ñañez, F. (2014). Diccionario de investigación científica cualitativa y cuantitativa. Concytec.

Sandín, M. P. (2003). Investigación cualitativa en educación. Fundamentos y tradiciones. McGrawHill.

Siemens, G. (2010). Conociendo el conocimiento. Ediciones Nodos Ele. https://bit.ly/3phJoqu

Stenhouse, L. (2007). La investigación como base de la enseñanza (6 $6^{\mathrm{a}} \mathrm{ed}$.). Morata.

Tobón, S. (2013). Metodología de gestión curricular: Una perspectiva socioformativa. Trillas.

Tobón, S. (2014). Currículo y ciclos propedeúticos desde la socioformación: Hacia un sistema flexible y sistémico. Trillas.

Tobón, S. (2015). Formación integral y competencias. Macro.

Tobón, S. (2017). Ejes esenciales de la sociedad del conocimiento y la socioformación. Kresearch.

Tobón, S. (2019). ¿Cómo lograr clases motivantes y participativas? Un enfoque socioformativo. Kresearch.

Espiral. Cuadernos del Profesorado | ISSN 1988-7701 | 2022, 15(30), 24-37 
Tobón, S., Cardona, S., Vélez, J., \& López, J. (2015). Proyectos formativos y desarrollo del talento humano para la sociedad del conocimiento. Revista Acción Pedagógica(24), 20-31. http://www.saber.ula.ve/handle/123456789/42127

Torres Ortiz, J. A., \& Barnabé Corrêa, T. H. (2020). Aspectos pedagógicos del conectivismo y su relación con redes sociales y ecologías del aprendizaje. Revista Brasileira de Educação, 25, 1-22. https://doi.org/10.1590/S1413-24782020250026

Trujillo, Z. C., Díaz, C., Travieso, M., Henríquez, D., \& Díaz, M. (2020). La transdisciplinariedad en el trabajo de los colectivos docentes del área clínica en Estomatología. Revista de Ciencias Médicas de Pinar del Río, 24(5), 1-11. https://bit.ly/3qibvoF

UNHEVAL. (2017). Modelo educativo. UNHEVAL.

Vázquez, J. M., Hernández, J. S., Vázquez, J., Juárez, L. G., \& Guzmán, C. E. (2017). El trabajo colaborativo y la socioformación: un camino hacia el conocimiento complejo. Revista Educación y Humanismo, 17(33), 334-356. http://dx.doi.org/10.17081/eduhum.19.33.2648

Zapata, J. S. (2015). El modelo y enfoque de formación por competencias en la Educación Superior: apuntes sobre sus fortalezas y debilidades. Revista Academia y Virtualidad, 8(2), 24-33. https://doi.org/10.18359/ravi.1420 